\title{
BMJ Open Investigating associations between physical activity and presenteeism: a scoping review protocol
}

\author{
Valérie Hervieux (10 , ${ }^{1,2,3}$ Caroline Biron, ${ }^{1,2,3}$ Justine Dima ${ }^{1,2}$
}

To cite: Hervieux V, Biron C, Dima J. Investigating associations between physical activity and presenteeism: a scoping review protocol. BMJ Open 2020;10:e040740. doi:10.1136/ bmjopen-2020-040740

- Prepublication history for this paper is available online. To view these files, please visit the journal online (http://dx.doi org/10.1136/bmjopen-2020040740).

Received 20 May 2020 Revised 21 October 2020 Accepted 15 November 2020

Check for updates

(C) Author(s) (or their employer(s)) 2020. Re-use permitted under CC BY-NC. No commercial re-use. See rights and permissions. Published by BMJ.

${ }^{1}$ Management, Laval University Faculty of Business Administration, Quebec, Quebec City, Canada

${ }^{2}$ Center of Expertise in

Occupational Health and Safety

Management, Laval University,

Quebec, Quebec City, Canada

${ }^{3}$ VITAM Research Center on

Sustainable Health, Quebec

Integrated University Health and

Social Services Center, Quebec,

Quebec City, Canada

Correspondence to

Valérie Hervieux;

valerie.hervieux.1@ulaval.ca

\begin{abstract}
Introduction Considering that physical activity plays a key role in the health of workers, a growing number of researchers are studying its relationship with various workplace outcomes, such as presenteeism. Numerous scientists recognise the relevance of further studying this relationship in order to improve our understanding of it. However, studies about the association between physical activity and presenteeism show some discrepancy in the results obtained. Disparity in the way of measuring presenteeism makes it even more challenging to compare results. In addition, it remains difficult to determine the optimal frequency, intensity, duration and type of physical activity to increase the productivity benefits of physical activity. In light of these issues, clarification through a scoping review of the literature on the subject is warranted.
\end{abstract}

Method and analysis A search strategy will be conducted in six scientific databases: MEDLINE, CINAHL, PsycINFO, ABI Inform Global, Web of Science and Business Source Premier. A screening process by two independent reviewers will lead to study selection. Quantitative and qualitative studies written in English about the relation between physical activity and presenteeism will be considered for inclusion. Data on the definition and measurement of presenteeism as well as the measurement of physical activity will be extracted. Additional data will be extracted to provide a descriptive overview of studies that have examined the relationship between presenteeism and physical activity.

Ethics and dissemination As this study will be based only on published studies, ethics approval is not required. Through the manner in which the included studies will be presented (categorised by their approach to presenteeism), this scoping review has the potential to improve our understanding of some of the inconsistencies observed in the literature. This review can also identify gaps in the existing evidence base and lead to new avenues of research.

\section{INTRODUCTION}

'Being physically active is one of the most important actions individuals of all ages can engage in to improve their health'. ${ }^{1}$ Physical activity is known to improve cardiorespiratory, metabolic, musculoskeletal, cognitive and mental health. ${ }^{12}$ In addition, a large body of research has shown that physical activity reduces the risk of cardiovascular disease, including coronary heart disease and ischaemic heart disease. ${ }^{3-5}$ However, the majority

\section{Strengths and limitations of this study}

This will be the first scoping review on the association between physical activity and presenteeism.

- Six scientific databases will be consulted.

- Data will be extracted and summarised in order to present what is currently known about this relation according to the definition of presenteeism.

of adults are not sufficiently active to achieve the health benefits of physical activity. ${ }^{1}$ Physical inactivity is considered the fourth leading risk factor for mortality worldwide and workplaces have been targeted as an ideal place to promote physical activity. ${ }^{6}$

In addition to these many health benefits, physical activity would also benefit organisations. For example, deterioration in employee health due to lack of physical activity, overweight and musculoskeletal pain is associated with presenteeism ${ }^{78}$ and absenteeism. ${ }^{7910}$ For organisations, employees with deteriorating health conditions (physical and mental) represent an increase in healthcare costs and a decrease in productivity. ${ }^{11}{ }^{12}$ As shown by the results of a recent study ${ }^{13}$ healthy behaviours, such as being physically active, may produce cost savings for the employer.

Considering that physical activity plays a key role in the health of workers, a growing number of researchers are investigating its relationship with various workplace outcomes, such as presenteeism. Numerous scientists recognise the relevance of further studying this relationship in order to improve our understanding of it. ${ }^{814-16}$

Studies that have examined the relationship between physical activity and presenteeism have used a variety of methodologies, making it difficult to compare study results. ${ }^{17-19}$ There is also some discrepancy in the results obtained. For example, some studies have shown a negative relationship between physical activity and presenteeism, ${ }^{2021}$ while others have revealed no significant relationship. ${ }^{22}{ }^{23}$ It is therefore 
essential to interpret these results with caution given the lack of consistency in the results, ${ }^{17}$ the variability in the methods used in these studies (eg, the many ways of measuring presenteeism and considering a physically active vs inactive individual ${ }^{15}$ and sometimes the lack of scientific rigour (eg, uncontrolled analyses for healthrelated variables).$^{24}$

One of the important findings of the review on the impact of physical activity on presenteeism ${ }^{15}$ is the wide variation in how studies conceptualise and evaluate presenteeism. The authors note that given these issues, their review is limited by the fact that the keywords used in the initial search may not have found all articles of interest in the literature. The issues surrounding the definition and measurement of presenteeism were recently raised in a position paper written by a group of researchers interested in the presenteeism..$^{25}$ Moreover, Navarro and his collaborators ${ }^{26}$ also stress the importance of comparing the results of studies on presenteeism with respect to the definition and conception of presenteeism, otherwise there is a risk that two completely different situations will be studied.

In addition, it remains difficult to determine the optimal frequency, intensity, duration and type of physical activity to increase the productivity benefits of physical activity. ${ }^{15} 27$ In light of these issues, clarification through a scoping review of the literature on the subject is warranted. A scoping review is of particular use when a body of literature exhibits a large, complex and heterogeneous nature ${ }^{28}$ as have this field of research. ${ }^{15}$

The study will consider the existing literature of the relation between physical activity and presenteeism. Publication bias will be limited through the use of comprehensive literature searching and specific inclusion criteria. In order to broadly cover and highlight what the literature reveals on the subject, we are not going to limit ourselves to a single understanding of presenteeism. Thus, keywords representing the various currents of research on presenteeism will be used. As a wide variety of measures of presenteeism are noted, ${ }^{29}$ the studies included will not be limited to some tool measurements or a tool explicitly derived from it (eg, translation of the tool). A scoping review will enable a better understanding of the strengths and limitations of the evidence to date and provide directions for future research. ${ }^{30}$

The aim of this study is to scope the literature on what is currently known between physical activity and presenteeism and answer the following questions:

1. What is known from the existing literature about the associations between physical activity and presenteeism over the past 20 years (2000-2020)?

2. How is physical activity measured in studies and in what context (ie, domains and intensity)?

3. How is presenteeism conceptualised and measured in those studies?

4. What do the results of the studies included in the review show?

\section{METHODS}

We will conduct this study using the framework of Arksey and O'Malley ${ }^{31}$ supplemented by recommendations and clarifications on advancing the methodology for scoping reviews. ${ }^{28} 32$

\section{Identifying relevant studies}

This search strategy has been validated by one experienced researcher $(\mathrm{CB})$ and one reference librarian. The identification of the relevant studies will be an iterative process that involved pre-testing many different combinations of subject headings, search terms and synonyms, to achieve the highest level of coverage. The primary searches will be conducted in six databases (MEDLINE via Ovid, CINAHL via EBSCOhost, PsycINFO via Ovid, ABI Inform Global via ProQuest, Web of Science and Business Source Premier via EBSCOhost) to identify peerreviewed articles.

Additional studies will be identified by scanning the reference lists of suitable studies and in personal collections. Experts in presenteeism will also be contacted through a list of researchers who participated in the small group meeting on presenteeism of the European Association for Work and Organizational Psychology. The start date of searches was set to 2000 to match the beginning of the interest in presenteeism in the scientific literature. ${ }^{33}$

Table 1 presents the search terms and the research queries that will be used on MEDLINE (Ovid).

\section{Study selection}

All search results will be downloaded and imported into a reference management software program (EndNote, V.X9) and then into the software Covidence to facilitate our process of screening for inclusion. The inclusion and exclusion criteria will be applied by two reviewers (VH and JD) to all potential studies at the title and abstract level. Where there is insufficient information to make an informed decision regarding a studies inclusion, then relevance to the next stage of the review process (full text assessment) will be assumed. The two reviewers will work independently using the inclusion criteria and meet to discuss any challenges or uncertainties related to study selection and to go back and refine the search strategy if needed. The remaining articles will be full text screened by VH and JD.

To be included in this review, studies will be required to be written in English. Studies that report new empirical data using a quantitative or qualitative design on association between physical activity and presenteeism will be included. The studies using health risk assessment, which measure health behaviours (such as physical activity), but not exclusively, will also be included. Studies that have some kind of physical activity (structured or not, during leisure-time or as a mean of transportation and so on) and to which the other criteria apply will be included. Studies dealing with non-working age populations such as elderly or children will be excluded. 


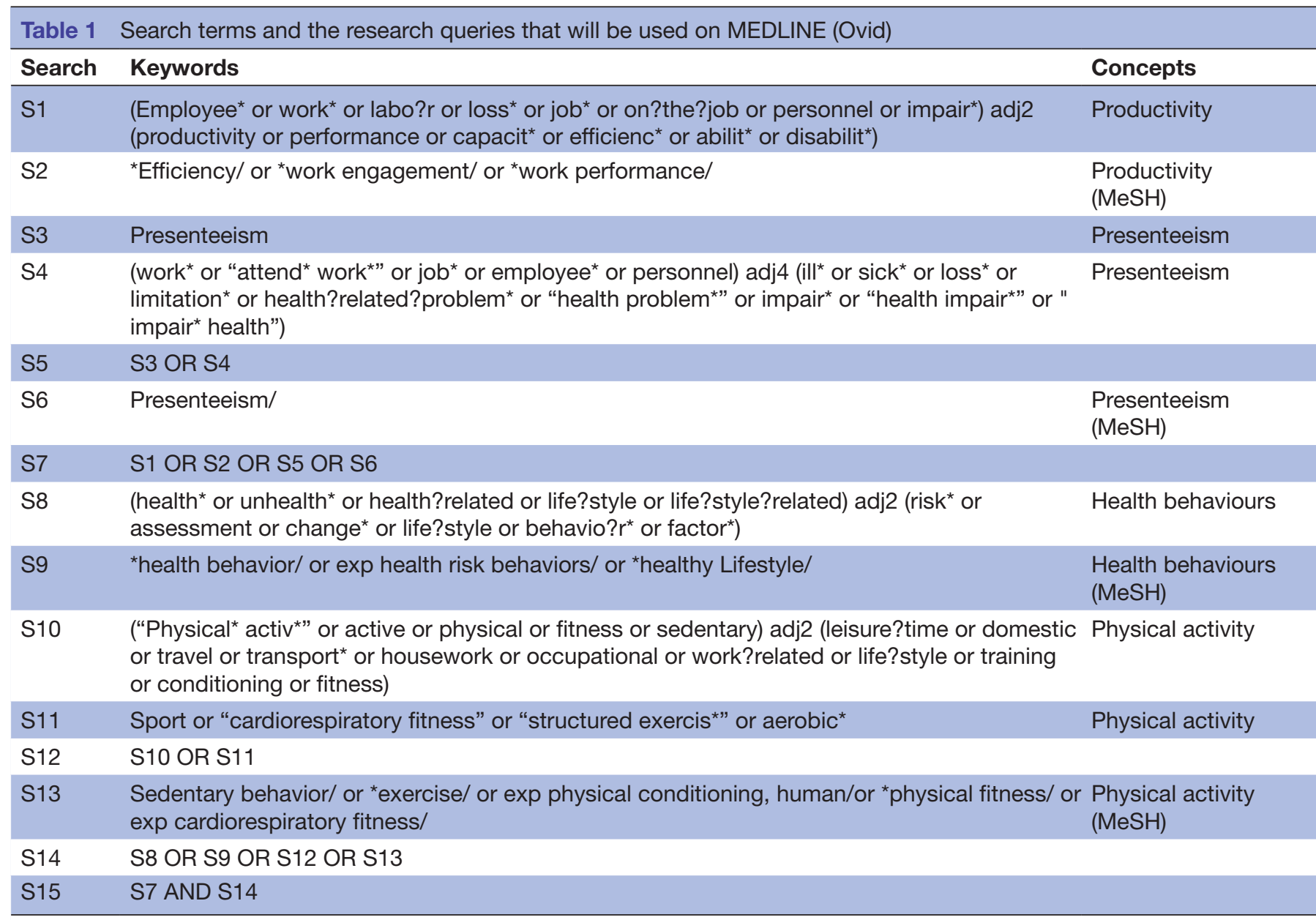

$\mathrm{MeSH}$, Medical Subject Headings.

\section{RESULTS}

\section{Data extraction}

Data will be extracted by one reviewer $(\mathrm{VH})$, and a random subset of at least $25 \%$ or the selected studies will be checked by another reviewer (JD) to verify repeatability and accuracy. To answer research questions \#2 and \#3 of this study, data on the definition and measurement of presenteeism as well as the measurement of physical activity will be extracted. To answer question \#4, the important results of the included studies will also be described. Finally, additional data will be extracted to provide a descriptive overview of studies that have examined the relationship between presenteeism and physical activity (author(s), year of publication, study location, study design, study populations).

\section{Data synthesis}

The results will be categorised according to the understanding of presenteeism of the studies to give a better idea of how this phenomenon is studied in relation to physical activity. This will help to address one of the issues raised by Navarro and colleagues, ${ }^{26}$ namely the difficulty of comparing studies since they do not view presenteeism in the same way. This will allow us to describe what we know about the relationship and answer question \#1 of this study.

\section{DISSEMINATION}

The results will be published in a peer-reviewed journal in the field of organisational health. The results of this review will be important for theoretical reasons and, more generally, knowledge advancement. First, through the manner in which the included studies are presented (categorised by their approach to presenteeism), this scoping review has the potential to improve our understanding of some of the inconsistencies observed in the literature. It will be easier to compare the results since it will be possible to quickly distinguish studies by the way presenteeism is defined and measured. As mentioned by other researchers, ${ }^{15}{ }^{26}$ the heterogeneity in the ways in which presenteeism is measured also limits the interpretation of the results of empirical studies. Second, the results of this review may contribute to the expansion of current literature on physical activity and presenteeism by including the domains of physical activity. Leisuretime physical activity has been largely investigated in relation with presenteeism, but little is known about 
the other domains (eg, transportation, occupational, domestic). The underlying mechanisms of how physical activity may contribute to presenteeism are as well very little documented. Finally, this review can identify gaps in the existing evidence base and lead to new avenues of research. For example, physical activity could be studied as an individual resource that promotes a more functional type of presenteeism. Karanika-Murray and Biron ${ }^{34}$ recently proposed a typology that considers presenteeism as an adaptive behaviour aimed at meeting work and performance demands during impaired capacity owing to ill-health (p.6). Physical activity could be an important resource used by employees to balance the demands of performance and health. This has important implications for interventions given that organisations can promote physical activity in the workplace by providing access to facilities, offering activities during work hours and promoting healthy lifestyle.

Acknowledgements We thank Louise Leblanc for her advice on this protocol and the reviewers and editor of the journal for their relevant comments of the first draft of this paper.

Contributors $\mathrm{VH}$ is the principal investigator of the study. $\mathrm{VH}$ planned and designed this study protocol and wrote the first draft. CB supervised the writing of the manuscript and brings expertise in presenteeism. All the authors revised and approved the final version of this manuscript. JD will also be the second reviewer.

Funding This protocol was supported by the Social Sciences and Humanities Research Council of Canada (the grant number\# 435-2018-0603).

Competing interests None declared.

Patient and public involvement Patients and/or the public were not involved in the design, or conduct, or reporting, or dissemination plans of this research.

Patient consent for publication Not required.

Provenance and peer review Not commissioned; externally peer reviewed.

Open access This is an open access article distributed in accordance with the Creative Commons Attribution Non Commercial (CC BY-NC 4.0) license, which permits others to distribute, remix, adapt, build upon this work non-commercially, and license their derivative works on different terms, provided the original work is properly cited, appropriate credit is given, any changes made indicated, and the use is non-commercial. See: http://creativecommons.org/licenses/by-nc/4.0/.

\section{ORCID iD}

Valérie Hervieux http://orcid.org/0000-0001-8003-2560

\section{REFERENCES}

1 Piercy KL, Troiano RP, Ballard RM, et al. The physical activity guidelines for Americans. JAMA 2018;320:2020-8.

2 Haskell WL, Blair SN, Hill JO. Physical activity: health outcomes and importance for public health policy. Prev Med 2009;49:280-2.

3 KohlHW. Physical activity and cardiovascular disease: evidence for a dose response. Med Sci Sports Exerc 2001;33:S472-83.

$4 \mathrm{Kyu} \mathrm{HH}$, Bachman VF, Alexander LT, et al. Physical activity and risk of breast cancer, colon cancer, diabetes, ischemic heart disease, and ischemic stroke events: systematic review and dose-response meta-analysis for the global burden of disease study 2013. BMJ 2016;354:i3857.

5 Miles L. Physical activity and health. Nutr Bull 2007;32:314-63.

6 WHO. Recommandations mondiales sur l'activité physique pour La santé 2010.

7 Pronk NP, Martinson B, Kessler RC, et al. The association between work performance and physical activity, cardiorespiratory fitness, and obesity. J Occup Environ Med 2004;46:19-25.

8 Schultz AB, Edington DW, Health E. Employee health and presenteeism: a systematic review. J Occup Rehabil 2007;17:547-79.
9 Jørgensen MB, Faber A, Hansen JV, et al. Effects on musculoskeletal pain, work ability and sickness absence in a 1-year randomised controlled trial among cleaners. BMC Public Health 2011;11:840.

10 McDonald M, DiBonaventura MdaCosta, Ullman S. Musculoskeletal pain in the workforce: the effects of back, arthritis, and fibromyalgia pain on quality of life and work productivity. J Occup Environ Med 2011:53:765-70.

11 Collins JJ, Baase CM, Sharda CE, et al. The assessment of chronic health conditions on work performance, absence, and total economic impact for employers. J Occup Environ Med 2005;47:547-57.

12 Lopez AD, Mathers CD, Ezzati M, et al. Global and regional burden of disease and risk factors, 2001: systematic analysis of population health data. Lancet 2006;367:1747-57.

13 Salmela J, Lahti J, Mauramo E, et al. Associations of changes in diet and leisure-time physical activity with employer's direct cost of short-term sickness absence. Eur J Sport Sci 2020;20:240-8.

14 LeCheminant JD, Merrill RM, Masterson T. Health behaviors and work-related outcomes among school employees. Am J Health Behav 2015;39:345-51.

15 Brown HE, Gilson ND, Burton NW, et al. Does physical activity impact on presenteeism and other indicators of workplace wellbeing? Sports Med 2011;41:249-62.

16 Proper KI, Staal BJ, Hildebrandt VH, et al. Effectiveness of physical activity programs at worksites with respect to work-related outcomes. Scand J Work Environ Health 2002;28:75-84.

17 Fonseca VR, Nobre MRC, Pronk NP, et al. The association between physical activity, productivity, and health care utilization among employees in Brazil. J Occup Environ Med 2010;52:706-12.

18 Cash SW, Beresford SAA, Henderson JA, et al. Dietary and physical activity behaviours related to obesity-specific quality of life and work productivity: baseline results from a worksite trial. $\mathrm{Br} J$ Nutr 2012;108:1134-42.

19 Block G, Sternfeld B, Block CH, et al. Development of alive! (a lifestyle intervention via Email), and its effect on health-related quality of life, presenteeism, and other behavioral outcomes: randomized controlled trial. J Med Internet Res 2008;10:e43.

20 Katz AS, Pronk NP, Lowry M. The association between optimal lifestyle-related health behaviors and employee productivity. J Occup Environ Med 2014;56:708-13.

21 Walker TJ, Tullar JM, Diamond PM, et al. The longitudinal relation between self-reported physical activity and presenteeism. Prev Med 2017:102:120-6.

22 Musich S, Hook D, Baaner S, et al. The association of corporate work environment factors, health risks, and medical conditions with presenteeism among Australian employees. Am J Health Promot 2006;21:127-36.

23 Gram B, Holtermann A, Bültmann U, et al. Does an exercise intervention improving aerobic capacity among construction workers also improve musculoskeletal pain, work ability, productivity, perceived physical exertion, and sick leave?: a randomized controlled trial. J Occup Environ Med 2012;54:1520-6.

24 Guertler D, Vandelanotte C, Short C, et al. The association between physical activity, sitting time, sleep duration, and sleep quality as correlates of presenteeism. J Occup Environ Med 2015;57:321-8.

25 Ruhle SA, Breitsohl H, Aboagye E, et al. "To work, or not to work, that is the question" - Recent trends and avenues for research on presenteeism. Eur J Work Organ Psychol 2020;29:344-63.

26 Navarro A, Salas-Nicás S, Llorens C, et al. Sickness presenteeism: are we sure about what we are studying? A research based on a literature review and an empirical illustration. $\mathrm{Am} J$ Ind Med 2019;62:580-9.

27 Walker TJ, Tullar JM, Diamond PM, et al. The relation of combined aerobic and muscle-strengthening physical activities with presenteeism. J Phys Act Health 2017;14:893-8.

28 Peters MDJ, Godfrey CM, Khalil H, et al. Guidance for conducting systematic scoping reviews. Int J Evid Based Healthc 2015;13:141-6.

29 Mattke S, Balakrishnan A, Bergamo G, et al. A review of methods to measure health-related productivity loss. Am J Manag Care 2007;13:211.

30 Pham MT, Rajić A, Greig JD, et al. A scoping review of scoping reviews: advancing the approach and enhancing the consistency. Res Synth Methods 2014;5:371-85.

31 Arksey H, O'Malley L. Scoping studies: towards a methodological framework. Int J Soc Res Methodol 2005;8:19-32.

32 Levac D, Colquhoun H, O'Brien KK. Scoping studies: advancing the methodology. Implement Sci 2010;5:69.

33 Johns G. Presenteeism in the workplace: a review and research agenda. J Organ Behav 2010;31:519-42.

34 Karanika-Murray M, Biron C. The health-performance framework of presenteeism: towards understanding an adaptive behaviour. Human Relations 2020;73:242-61. 